\title{
Analysis of Thematic Classified Aerial Images Trough Multispectral and LIDAR Data
}

\author{
A. Arquero and E. Martínez
}

\begin{abstract}
The application of thematic maps obtained through the classification of remote images needs the obtained products with an optimal accuracy. The registered images from the airplanes display a very satisfactory spatial resolution, but the classical methods of thematic classification not always give better results than when the registered data from satellite are used. In order to improve these results of classification, in this work, the LIDAR sensor data from first return (Light Detection And Ranging) registered simultaneously with the spectral sensor data from airborne are jointly used. The final results of the thematic classification of the scene object of study have been obtained, quantified and discussed with and without LIDAR data, after applying different methods: Maximum Likehood Classification, Support Vector Machine with four different functions kernel and Isodata clustering algorithm (ML, SVM-L, SVM-P, SVM-RBF, SVM-S, Isodata). The best results are obtained for SVM with Sigmoide kernel. These allow the correlation with others different physical parameters with great interest like Manning hydraulic coefficient, for their incorporation in a GIS and their application in hydraulic modelling.
\end{abstract}

Keywords - image classification, aerial photography, LIDAR (Light Detection And Ranging), hydraulic modelling.

\section{INTRODUCCIÓN}

$\mathrm{L}^{\mathrm{s}}$ AS fuentes de datos mas comunes en Teledetección, incluyen tanto a la fotografía aérea, como a sensores espectrales y radar soportados en avión y satélite. Una de las tareas más frecuentes y de gran interés, que se realiza en el procesado de imágenes registradas desde los sensores remotos es la clasificación temática. La buena resolución espacial de los sensores trasportados en aviones, hacen que la fotografía aérea se presente como una buena tecnología para la obtención de mapas temáticos. Sin embargo, los resultados obtenidos en la aplicación de los clasificadores clásicamente utilizados, no dan siempre resultados satisfactorios en la buena discriminación de las coberturas terrestres. La disposición de datos LIDAR (Light Detection And Ranging) representa una nueva posibilidad para optimizar la clasificación temática de dichas coberturas. En el caso de cubiertas vegetales, se han utilizado estos sensores (LIDAR) para mejorar su clasificación, ya que se puede medir directamente tanto la localización vertical como la distribución horizontal de elementos de la cubierta vegetal, usando longitudes de onda en el Infrarrojo (IR) o Infrarrojo Cercano (NIR) [1].

En las tareas de clasificación es importante disponer de

A. A. Hidalgo, Universidad Politecnica de Madrid, Madrid, Spain, aarquero@fi.upm.es

E. M. Izquierdo, Universidad Politecnica de Madrid, Madrid, Spain, emartinez@fi.upm.es datos de diferentes fuentes de información, para optimizar los resultados. A partir de datos LIDAR se pueden generar Modelos Digitales de Elevaciones (DEM) con gran precisión para usos topográficos y para caracterizar sedimentos en erosiones de ríos [2]. Lee y Shan [3] han usado datos de elevación LIDAR aéreos junto con imágenes multiespectrales IKONOS sobre una superficie costera para identificar seis tipos de clases de ocupación terrestre (carretera, agua, humedales, edificaciones, árboles y arena) y concluyen que la inclusión de datos LIDAR es beneficiosa para la separación de las clases, que por otra parte presentan similares respuestas espectrales. Los errores globales de clasificación, particularmente los falsos positivos se reducen por encima del $50 \%$. Los resultados de clasificación con LIDAR mostraron una distribución más realista y homogénea de las formas geométricas.

El uso de sensores LIDAR está aumentando para aplicaciones relacionadas con el estudio de bosques. Bork y $\mathrm{Su}$ [4] realizaron un estudio comparativo de los resultados obtenidos en la clasificación espacialmente compleja de una reserva natural en Canadá. Utilizaron once clases de vegetación (tres con mayoría de bosque caduco y ocho de otros tipos de vegetación). Con los datos LIDAR, el sistema para tres clases generó un DEM (modelo digital de elevación) de vegetación que incluía la información derivada de la altura. El estudio para las ocho clases restantes incorporó las características topográficas de la escena. Aplicaron dos métodos de clasificación al mosaico original de la imagen digital; a una imagen color híbrida compuesta por relaciones entre bandas, y a una imagen IHS (Intensity-Hue-Saturation) usando técnicas de entrenamiento supervisadas. Sin embargo no fueron utilizados los datos LIDAR incorporándolos como una nueva banda para la clasificación temática.

En algunos estudios se usan los datos LIDAR para solucionar problemas de clasificación, concretamente en casos en los que se investiga un número reducido de clases temáticas, como es el caso de la discriminación entre árboles caducos y coníferas [5]-[7]. Brennan y Webster [5] en su estudio utilizan cuatro capas (intensidad media, altura normalizada, modelo digital de superficies, MDS y retornos múltiples LIDAR) para diferenciar entre diez estructuras terrestres.

La utilización de datos hiperespectrales y LIDAR para la clasificación de áreas urbanas [8] se ha centrado en la segmentación de la escena mediante los datos LIDAR y una clasificación de las regiones resultantes a partir de los datos hiperespectrales. 
Otros estudios explotan los datos LIDAR en una fase de preprocesado, usando un modelo digital del terreno [9] derivado del LIDAR en la fase de corrección geométrica de imágenes hiperespectrales. La mayoría de los estudios realizados usando datos espectrales y LIDAR no llevan a cabo la integración de ambos tipos de datos, sino que utilizan estas fuentes de información de forma separada para las diferentes fases del proceso en que están implicados. Así, en el momento actual, solo unos pocos investigadores han llevado a cabo el diseño de sistemas avanzados de clasificación capaces de explotar adecuadamente la información complementaria presente en estos datos y la posibilidad de usar conjuntamente datos LIDAR y espectrales para la clasificación de áreas forestales complejas donde hay presencia de varias especies arbóreas.

Recientemente Dalponte et al. [10] proponen un sistema avanzado de clasificación temática usando datos LIDAR e hiperespectrales, estudiando la importancia de los primeros cuando se fusionan con imágenes hiperespectrales para resolver problemas de clasificación compleja de bosques y proporcionan la posibilidad de estimar parámetros característicos de los bosques.

Bartels y Wei [11] aplican métodos de clasificación de máxima probabilidad con datos LIDAR fusionados con bandas espectrales co-registradas, extrayendo tipos de terreno que incluyen edificaciones, vegetación y suelo con rangos de elevaciones pequeñas para una superficie urbana de tamaño reducido $\left(800 \mathrm{~m}^{2}\right)$. También se están usando estos datos aéreos para realizar clasificación de cubiertas terrestres basadas en objetos [12].

En los últimos trabajos presentados [13] se muestra la utilidad de la integración de datos LIDAR con imágenes multiespectrales QuickBird (QB) para generar modelos de mapas de combustibilidad de bosques. La clasificación supervisada de la imagen se llevó a cabo usando reglas de decisión paramétricas como Máxima Probabilidad y reglas de decisión de distancia de Mahalanobis, entre otras con la imagen multiespectral y con el conjunto de LIDAR/ multiespectral.

En este trabajo se trata de realizar un estudio comparativo entre métodos como ML de clasificación supervisada ampliamente utilizado, Isodata con semillas de agrupamiento y los basados en SVM; analizando la bondad de la clasificación y las diferentes características asociadas a la discriminación de las clases temáticas, para imágenes aéreas multiespectrales, unidas o no con datos LIDAR. Se utiliza un MDS obtenido a partir de los datos del primer retorno LIDAR y la imagen multiespectral de cuatro bandas adquirida simultáneamente con estos datos, para discriminar 16 tipos de cubiertas terrestres. Se han encontrado clases temáticas diversas que incluyen la presencia de agua en río, vegetación arbolada y de cultivo, carreteras en diferentes alturas por la presencia de puentes, suelos con distinta cobertura de vegetación y estructuras de edificaciones urbanas. Algunas de estas cubiertas son fácilmente discriminadas por su firma espectral característica en la imagen multiespectral y de ellas se podrían obtener las áreas de entrenamiento para la clasificación mediante el uso de dispersogramas entre bandas [14] y consideraciones estadísticas [15]. Sin embargo, hay otras que solamente se pueden diferenciar por su dato de altura procedente de la respuesta LIDAR.

Los métodos tradicionales de clasificación incluyen clasificadores clásicos como el de Máxima Verosimilitud (Maximum Likehood) y Mínima Distancia (Minimum Distance). Recientemente las metodologías de clasificación que incluyen SVM (Support Vector Machine) focalizan la atención de los investigadores del mundo de la Teledetección [16]. Los métodos SVM tienen sus raíces en la Teoría del Aprendizaje Estadístico [17]. Estos pertenecen al grupo de clasificadores no paramétricos, considerados de los más robustos y están a la cabeza de los métodos de clasificación por permitir construir fronteras de decisión flexibles, y por su buena capacidad de generalización. El método SVM permite abordar de manera general la resolución de problemas de clasificación y de regresión. Se transforma el conjunto de vectores de entrada o patrones $n$-dimensionales de entrenamiento en otro de vectores de una dimensión más alta en los que el problema pueda solucionarse linealmente. Se trata de un clasificador que se puede entrenar con gran velocidad, permite construir hiperplanos óptimos de separación en problemas de clasificación y tiene capacidad para trabajar con datos de dimensión muy alta. Cuando las clases no son linealmente separables transforma los vectores de entrada en vectores de mayor dimensión para conseguir linearidad. SVM se trasforma en un clasificador lineal a partir de la utilización de kernels no lineales. Otra de las características es que aproxima la probabilidad de que un patrón de entrada pertenezca o no a una clase, mediante funciones (relaciones) entre los datos de entrada. Así se trata de un método basado en funciones de kernel y la similitud entre los datos se expresa a través de la noción de kernel, definido según el problema a resolver. Mientras que en su forma más simple, se trata de un clasificador binario, se puede utilizar como clasificador multiclase por combinación de múltiples clasificadores binarios, creando un clasificador binario por cada par de clases. Para obtener un clasificador multiclase, varios investigadores han propuesto algunas aproximaciones como crear varios clasificadores binarios en serie [18], o una organización de una estructura jerárquica [19].

\section{ADQUISICIÓN Y CARACTERÍSTICAS DE LOS DATOS}

El área de estudio seleccionada es una escena correspondiente a una zona de la ciudad de Zaragoza (España) lindante con parcelas rústicas parcialmente cultivadas, Fig. 1, cuyo centro tiene unas coordenadas geográficas UTM 678598,96 E y 4613320,06 N. La dimensión es de 1363×1363 píxeles con un tamaño de $0,5 \mathrm{~m}$ de lado. Los datos multiespectrales y LIDAR, Fig. 2, se adquirieron en Agosto de 2006.

La imagen aérea multibanda presenta tres bandas en el rango visible, $\mathrm{B}(0,40-0,58 \mu \mathrm{m}), \mathrm{G}(0,50-0,65 \mu \mathrm{m})$ y $\mathrm{R}(0,59-$ 
$0,68 \mu \mathrm{m})$ y una en el NIR $(0,74-0,85 \mu \mathrm{m})$ del espectro electromagnético. La resolución radiométrica es de 12 bits. Ha sido registrada por una cámara de área aerotransportada DMC (Digital Mapping Camera) de Z/I Imaging [20], que contiene 4 módulos sensores CCD matriciales pancromáticos de $7.000 \times 4.000$ píxeles (28 megapíxeles) y 4 módulos sensores CCD matriciales multiespectrales de $2.000 \times 3.000$ píxeles $(6$ megapíxeles), con un tamaño del píxel de 12 micras y precisión geométrica de 0,1 micra, que es un $77 \%$ más sensible a la luz que el de 9 micras. La distancia focal del pancromático es de $120 \mathrm{~mm}$ y la multiespectral de $25 \mathrm{~mm}$. La velocidad del disparador es variable de $1 / 50$ a 1/300 s con apertura de $\mathrm{f} / 4$ a f/22. Su resolución geométrica puede variar entre $0,04 \mathrm{~m}$ o $1 \mathrm{~m}$ de tamaño de píxel sobre el terreno según la altura de vuelo. Presenta compensación FMC (Forward

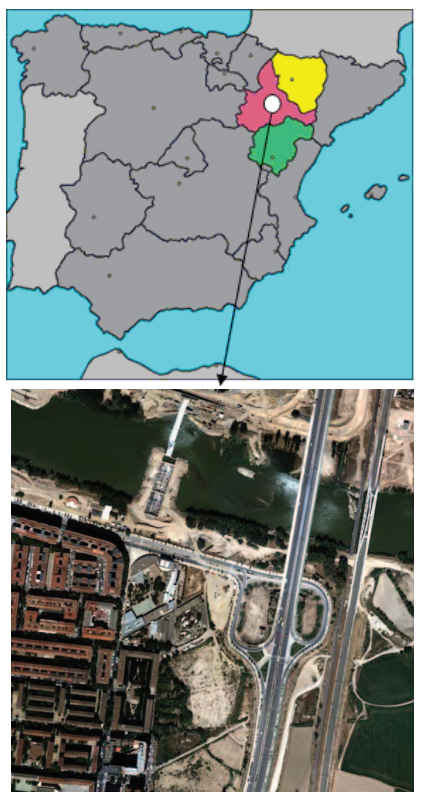

Figura 1. Localización del área estudiada y orto-imagen RGB.

Motion Correction) electrónica TDI (Time Delay Integrated) sin limitaciones por mecánica. También es importante la incorporación de sistemas de almacenamiento masivo en vuelo FDS (Flight Data Storage). Presenta disposición hacia el nadir en la aeronave.

Los datos LIDAR se han adquirido desde la misma aeronave, con un sistema ALS50-II de Leica, que presenta una frecuencia de pulso máxima de $85 \mathrm{kHz}$ (85.000 puntos por segundo) y con una frecuencia máxima de barrido dependiente del FOV (Field Of View): $36 \mathrm{~Hz}$ para FOV de $40^{\circ}$ y $24 \mathrm{~Hz}$ para $75^{\circ}$. Presenta 4 retornos. Puede operar entre $200 \mathrm{~m}$ y $4000 \mathrm{~m}$ sobre el terreno. Su campo de visión (FOV) puede variar de $10^{\circ}$ a $75^{\circ}$ y presenta compensación automática de Roll (omega), siendo sus capturas de intensidades de una por eco. Se usó un sistema GPS/INS integrado en tiempo real con la adquisición de todos los datos. Las imágenes multibanda fueron proyectadas en el sistema de coordenadas UTM.

En este trabajo se ha utilizado la imagen aérea multibanda

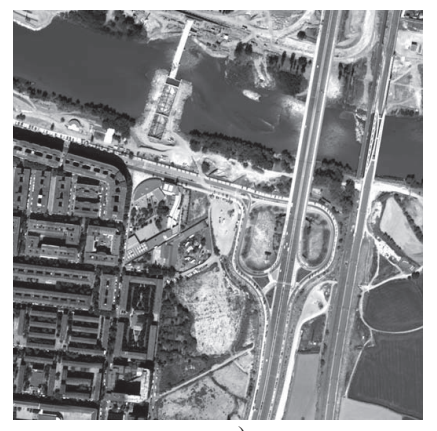

a)



Figura 2. a) Banda $\mathrm{G}$ de la imagen aérea multiespectral. b) Banda 5 con MDS de LIDAR.

y el primero de los retornos LIDAR. No se han realizado modificaciones a la imagen digital multibanda como las que se aplican en otros trabajos de la bibliografía [4] referidas a la eliminación de sombras. En otras investigaciones [21] se presentan diagramas de flujo, donde todos los datos se normalizan en un único MDS, a partir del cual se realiza un estudio sobre tipos de árboles. Sin embargo, en el presente trabajo se unen los datos espectrales originales de la imagen aérea multibanda con el MDS procedente de los datos LIDAR co-registrados.

Para su utilización en los procesos de clasificación temática, los datos de la capa de la imagen denominada B5, son los originales, procedentes del LIDAR, reescalados adecuadamente con el fin de que ejercieran un efecto más discriminante, ya que los datos originales se encontraban en un rango de 48 niveles digitales diferentes.

\section{DEFINICIÓN DE LAS CLASES Y ARQUITECTURA DEL PROCESO}

Como ya se ha comentado tras la revisión bibliográfica, los estudios realizados utilizando imágenes multiespectrales y datos LIDAR simultáneamente, no ha sido muy habitual. Por un lado, los procesos de clasificación temática clásicos y supervisados, como el paramétrico de Máxima Verosimilitud (ML), han demostrado su eficacia para sensores como TM y ETM+ de Landsat, pero no tanto cuando el sensor presenta resoluciones espaciales mejores, como es el caso de imágenes QB, para las que hay que recurrir a otras técnicas de tratamiento de la imagen como son los procesos de fusión y llegar a un equilibrado entre las resoluciones espacial y espectral [22]. 
Por otra parte, los procesos de clasificación temática clásicos y no supervisados, como el Isodata original, pueden resultar demasiado aleatorios en su búsqueda de centroides [23] para realizar su clasificación. Por esta razón genera determinadas clases cuya representatividad en la imagen es mínima, despreciando otras que pueden ser observadas con un solo análisis visual de la imagen aérea. Estos comportamientos de los diferentes clasificadores se muestran en los resultados obtenidos.

Respecto a la variedad de clases temáticas presentes en la escena objeto de estudio, hay que resaltar la presencia de cubiertas tanto de vegetación y suelos de origen natural como otras introducidas por el hombre.

En la Fig. 3 se define la arquitectura utilizada en el proceso de este trabajo. Primeramente, se realiza el mosaico de los datos de la cámara digital que engloba la escena a estudiar. Por otro lado, los datos LIDAR de superficies, obtenidos a partir del primer retorno $(1 \mathrm{~m})$, se adaptan a un tamaño de píxel igual al de la imagen $(0,5 \mathrm{~m})$, se recorta para ajustar al tamaño de la imagen multiespectral y se reescala radiométricamente. El siguiente paso es realizar una unión de todos los datos, 4 bandas espectrales (B, G, R, NIR) y una nueva banda (B5) de LIDAR. Así la imagen se compone de 5 bandas de datos.

A la imagen final, con y sin datos LIDAR, se han aplicado seis métodos de clasificación, cinco supervisados y por tanto con una selección previa de áreas de entrenamiento de las clases objeto de clasificación y otro no supervisado (Isodata) que elige aleatoriamente los centroides de las clases de entrenamiento. El objetivo ha sido comprobar el resultado temático de la clasificación de imágenes aéreas cuando se dispone de información LIDAR.

\section{Resultados}

Para aplicar los métodos de clasificación supervisados se han elegido áreas de entrenamiento correspondientes a 16 clases temáticas distinguibles en la escena. Las características de estas cubiertas terrestres son las siguientes:

Cultivo: Terreno rústico cultivado, con altura baja.

Suelo 1: Suelo con vegetación escasa y altura baja.

Suelo 2: Suelo en barbecho claro y altura baja.

Suelo 3: Suelo en barbecho más oscuro y altura baja.

Suelo 4: Suelo removido, muy reflectante y altura baja.

Urbano 1: Suelo ocupado por edificaciones más altas.

Urbano 2: Suelo ocupado por edificaciones un poco más bajas que las de urbano 1 .

Urbano 3: Suelo ocupado por edificaciones de altura media.

Urbano 4: Suelo ocupado por edificaciones muy reflectantes

y de altura media.

Veg. 1: Arbolado verde y de altura media-alta.

Veg. 2: Arbolado de color verde menos intenso y de altura media.

Vías 1: Carreteras y vías de tren en altura mayor (por la existencia de puentes) y mayor reflectancia.
Vías 2: Carreteras y vías de tren de menor reflectancia y altura.

Río: Río Ebro a su paso por la ciudad de Zaragoza, profundo en su mayor parte y de la altura menor.

Sombra en el río: Sombra producida la inclinación del sol sobre los árboles cercanos a la orilla.

Sombra en el suelo: Sombra producida por los edificios y árboles sobre el entorno, distinguida por el dato LIDAR.

Para evaluar la precisión de cada clasificación se usan métodos estadísticos [24]. Las matrices de error se construyen comparando los resultados con áreas test supervisadas en campo. Se calculan a partir de ellas, la precisión global y el índice kappa (Tabla I). Los resultados indican que el método SVM con función Sigmoid (con LIDAR) es el que ofrece una clasificación más de acorde con los datos test de verdad de campo por tener un valor mayor de los parámetros de precisión. Por tanto se consideran los valores de las firmas medias resultantes de esta clasificación en la Tabla II,


Irragen final (5 bandas)

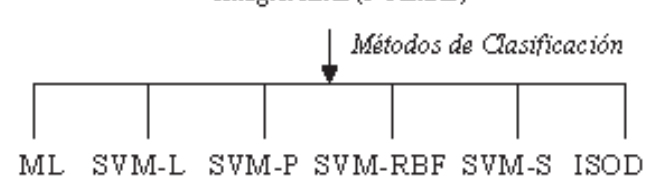

Figura 3. Arquitectura de los procesos seguidos.

como los representativos de los tipos de cubiertas obtenidas.

TABLA I

PARÁMETROS DE ERROR: OVERALL ACCURACY (\%) Y COEFICIENTE KAPPA PARA LAS IMÁGENES CLASIFICADAS

\begin{tabular}{|l|c|c|c|c|}
\hline Escena & \multicolumn{2}{|c|}{ Con datos LIDAR } & \multicolumn{2}{c|}{ Sin datos LIDAR } \\
\hline Parámetros & $\mathrm{O}(\%)$ & $\mathrm{K}$ & $\mathrm{O}(\%)$ & $\mathrm{K}$ \\
\hline ML & 98,7013 & 0,9861 & 77,2727 & 0,7576 \\
\hline SVM L & 98,4848 & 0,9838 & 84,1991 & 0,8314 \\
\hline SVM P & 99,3506 & 0,9931 & 83,5498 & 0,8245 \\
\hline SVM RBF & 99,3506 & 0,9931 & 83,3333 & 0,8222 \\
\hline SVM Sigmoid & 100 & 1 & 74,8918 & 0,7321 \\
\hline Isodata & 65,5527 & 0,6282 & 60,2978 & 0,5731 \\
\hline
\end{tabular}


TABLA II

FIRMAS ESPECTRALES MEDIAS DE LAS CLASES TEMÁTICAS OBTENIDAS MEDIANTE EL MÉTODO SVM SIGMOID CON DATOS LIDAR

\begin{tabular}{|l|c|c|c|c|c|}
\hline CLASE & B1 & B2 & B3 & B4 & B5 \\
\hline Cultivo & 23,4 & 50,9 & 22,8 & 95,8 & 39,4 \\
\hline Suelo1 & 27,8 & 58,9 & 34,2 & 67,6 & 40,8 \\
\hline Suelo 2 & 62 & 135,4 & 82 & 92,2 & 45,1 \\
\hline Suelo3 & 46,3 & 100,8 & 61,7 & 76,9 & 44,8 \\
\hline Suelo 4 & 75,2 & 164,2 & 98,4 & 104,4 & 54 \\
\hline Urbano 1 & 32,7 & 73 & 55,4 & 66,1 & 147,7 \\
\hline Urbano 2 & 36,1 & 73,7 & 45,1 & 50 & 142,4 \\
\hline Urbano 3 & 45,1 & 96,6 & 58,5 & 70,6 & 76,6 \\
\hline Urbano 4 & 111,7 & 203,3 & 117,1 & 113,1 & 76,5 \\
\hline Veg. 1 & 22,4 & 47,9 & 26,3 & 61,3 & 110,7 \\
\hline Veg. 2 & 18,6 & 38,4 & 18,9 & 71,4 & 79,7 \\
\hline Vías 1 & 62,5 & 121,8 & 67 & 70 & 94,8 \\
\hline Vías 2 & 42,9 & 82,1 & 45,9 & 47,9 & 69,5 \\
\hline Río & 23,1 & 50,8 & 25,1 & 19,2 & 13,9 \\
\hline Sombra en río & 13,8 & 22,1 & 11,9 & 17,9 & 15,6 \\
\hline Sombra en suelo & 13,9 & 22,1 & 13 & 18,7 & 81,4 \\
\hline
\end{tabular}

Para los clasificadores supervisados, utilizando la imagen con datos LIDAR, se observan valores menores del $100 \%$ pero superiores al $80 \%$, en la precisión del productor y/o del usuario para las clases de Cultivo, Suelo 1, Veg. 1 y Veg. 2 (Tabla III del Apéndice).

Estas clases presentan firmas parecidas excepto en B4 (Cultivo y Suelo 1) y en B5 (Veg.1 y Veg. 2) como se puede apreciar en la Tabla IV del Apéndice.

Cuando se aplican los mismos métodos de clasificación a la imagen sin datos LIDAR, los resultados de precisión empeoran notablemente, tanto en las cubiertas citadas anteriormente como también para las Urbano 2, Vías 1 y Vías 2 , con algunos resultados sorprendentemente bajos (del orden del $6 \%)$.

Estos resultados indican claramente el gran beneficio que supone el uso de datos LIDAR para la precisión del mapa temático resultante de la clasificación.

Respecto a los resultados obtenidos al aplicar un clasificador no supervisado, como es el Isodata, son claramente peores, además de no reconocer la existencia de ciertas clases temáticas como es el Urbano 3 y la Sombra en río, los valores de precisión del $0 \%$ para Suelo 4 , Veg. 1, Vías 1 y Vías 2, cuando se usan datos LIDAR o las clases de Vías 2 y Sombra en río y con valores de $0 \%$ para Suelo 4, Urbano 2 y Veg. 2, cuando no se usan los datos LIDAR.

En la Fig. 4 se muestra el mejor, el peor resultado de las clasificaciones realizadas y el color asignado a las clases.

\section{CONCLUSIONES}

Como consecuencia de los resultados obtenidos se puede concluir que la utilización de datos de altura LIDAR de sensores aerotransportados, corregistrados con imágenes multiespectrales, es decisiva para obtener los mejores resultados de la clasificación temática de imágenes de alta resolución espacial y mediana resolución espectral, que de otra manera no se podrían conseguir. Se pueden discriminar clases temáticas espectralmente muy parecidas, pero de diferentes alturas, por ejemplo una carretera que se sitúa en diferentes alturas (Vías 1 y Vías 2), edificios de alturas diversas (Urbano 1-4), cubiertas de vegetación con altura baja
(Cultivo) de otras con altura mayor (arbolado, Veg. 1 y Veg. 2) y determinación de la existencia de diferentes tipos de sombras (Sombra en río y Sombra en suelo), así como su discriminación de la clase Río, con la menor respuesta espectral en las bandas 1-4, como se observa en los datos de la Tabla II.



a)

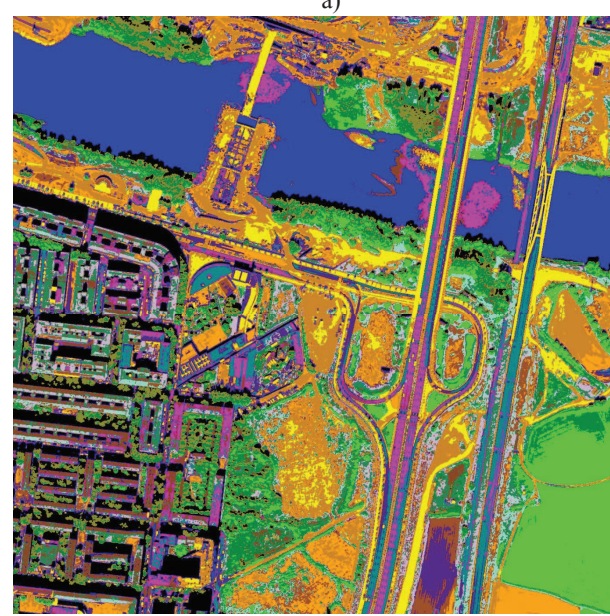

b)



c)

Figura 4. Ejemplos de: a) la mejor clasificación (SVM-Sigmoide); b) la peor (Isodata sin LIDAR) y c) los colores asignados a las clases temáticas.

Estos procesos de clasificación pueden ser usados para diversas aplicaciones, como pueden ser la detección de cambios en el uso del suelo. También pueden asociarse determinados parámetros correspondientes a las clases 
temáticas, como rugosidad o índice de Manning, para su incorporación en un SIG [25], que sean de utilidad en modelos hidráulicos.

\section{APÉNDICE: TABLAS DE RESULTADOS DE ESTADÍSTICAS COMPLEMENTARIAS}

En este Apéndice se incorpora la tabla comparativa de la precisión del productor y del usuario para los métodos de clasificación utilizados, correspondientes a los resultados cuando se utilizan datos LIDAR. También se muestra la tabla con las firmas espectrales medias para todas las clases y todos los métodos de clasificación aplicados.

TABLA III

PRECISIÓN DE PRODUCTOR (PP) (\%) Y DE USUARIO (PU) (\%) EN LAS CLASES TEMÁTICAS PARA LOS CLASIFICADORES APLICADOS A LA ESCENA CON LIDAR.

\begin{tabular}{|c|c|c|c|c|c|c|}
\hline \multirow[b]{2}{*}{ Clases } & \multicolumn{2}{|c|}{ ML } & \multicolumn{2}{|c|}{ SVM L } & \multicolumn{2}{|c|}{ SVM P } \\
\hline & PP & $\mathrm{PU}$ & $\mathrm{PP}$ & $\mathrm{PU}$ & PP & $\mathrm{PU}$ \\
\hline Cultivo & 85,7 & 100 & 71,4 & 100 & 85,7 & 100 \\
\hline Suelo 1 & 96 & 88,9 & 100 & 80,6 & 100 & 89,3 \\
\hline Suelo 2 & 100 & 100 & 100 & 100 & 100 & 100 \\
\hline Suelo 3 & 100 & 100 & 100 & 100 & 100 & 100 \\
\hline Suelo 4 & 100 & 100 & 100 & 100 & 100 & 100 \\
\hline Urbano 1 & 100 & 100 & 100 & 100 & 100 & 100 \\
\hline Urbano 2 & 100 & 100 & 100 & 100 & 100 & 100 \\
\hline Urbano 3 & 100 & 100 & 100 & 100 & 100 & 100 \\
\hline Urbano 4 & 100 & 100 & 100 & 100 & 100 & 100 \\
\hline Veg 1 & 100 & 95,7 & 100 & 97,8 & 100 & 100 \\
\hline Veg 2 & 93,1 & 100 & 96,5 & 100 & 100 & 100 \\
\hline Vias 1 & 100 & 100 & 100 & 100 & 100 & 100 \\
\hline Vias 2 & 100 & 100 & 100 & 100 & 100 & 100 \\
\hline Rio & 100 & 100 & 100 & 100 & 100 & 100 \\
\hline $\begin{array}{l}\text { Sombra } \\
\text { en rio }\end{array}$ & 100 & 100 & 100 & 100 & 100 & 100 \\
\hline $\begin{array}{l}\text { Sombra } \\
\text { en suelo }\end{array}$ & 100 & 100 & 100 & 100 & 100 & 100 \\
\hline \multirow[b]{2}{*}{ Clases } & \multicolumn{2}{|c|}{ SVM RBF } & \multicolumn{2}{|c|}{$\begin{array}{c}\text { SVM } \\
\text { Sigmoid }\end{array}$} & \multicolumn{2}{|c|}{ Isodata } \\
\hline & PP & PU & $\mathrm{PP}$ & $\mathrm{PU}$ & PP & $\mathrm{PU}$ \\
\hline Cultivo & 85,7 & 100 & 100 & 85,7 & 100 & 100 \\
\hline Suelo 1 & 100 & 89,3 & 100 & 100 & 89,3 & 100 \\
\hline Suelo 2 & 100 & 100 & 100 & 100 & 100 & 100 \\
\hline Suelo 3 & 100 & 100 & 100 & 100 & 100 & 100 \\
\hline Suelo 4 & 100 & 100 & 100 & 100 & 100 & 100 \\
\hline Urbano 1 & 100 & 100 & 100 & 100 & 100 & 100 \\
\hline Urbano 2 & 100 & 100 & 100 & 100 & 100 & 100 \\
\hline Urbano 3 & 100 & 100 & 100 & 100 & 100 & 100 \\
\hline Urbano 4 & 100 & 100 & 100 & 100 & 100 & 100 \\
\hline Veg 1 & 100 & 100 & 100 & 100 & 100 & 100 \\
\hline Veg 2 & 100 & 100 & 100 & 100 & 100 & 100 \\
\hline Vias 1 & 100 & 100 & 100 & 100 & 100 & 100 \\
\hline Vias 2 & 100 & 100 & 100 & 100 & 100 & 100 \\
\hline Rio & 100 & 100 & 100 & 100 & 100 & 100 \\
\hline $\begin{array}{l}\text { Sombra } \\
\text { en rio }\end{array}$ & 100 & 100 & 100 & 100 & 100 & 100 \\
\hline $\begin{array}{l}\text { Sombra } \\
\text { en suelo }\end{array}$ & 100 & 100 & 100 & 100 & 100 & 100 \\
\hline
\end{tabular}

TABLA IV

FIRMAS ESPECTRALES MEDIAS DE LAS CLASES TEMÁTICAS OBTENIDAS MEDIANTE TODOS LOS CLASIFICADORES CON DATOS LIDAR

\begin{tabular}{|c|c|c|c|c|c|c|}
\hline Método & Clase & B1 & B2 & B3 & B4 & B5 \\
\hline ML & Cultivo & 23,4 & 50,7 & 22,4 & 94,7 & 39,8 \\
\hline SVM-L & & 23,4 & 50,7 & 22,7 & 95,7 & 40,4 \\
\hline
\end{tabular}

\begin{tabular}{|c|c|c|c|c|c|c|}
\hline SVM-P & & 23,4 & 50,8 & 22,8 & 95,8 & 40,2 \\
\hline SVM-RBF & & 23,3 & 50,8 & 22,7 & 95,8 & 40,1 \\
\hline $\begin{array}{c}\text { SVM- } \\
\text { Sigmoid }\end{array}$ & & 23,4 & 50,9 & 22,8 & 95,8 & 39,4 \\
\hline Isodata & & 24 & 52 & 25 & 89,1 & 39,4 \\
\hline ML & Suelo1 & 35,2 & 77,3 & 47,3 & 76,7 & 45,5 \\
\hline SVM-L & & 27,5 & 58,1 & 33,5 & 67,5 & 41 \\
\hline SVM-P & & 27,9 & 59,2 & 34,3 & 68,3 & 40,5 \\
\hline SVM-RBF & & 27,9 & 59,2 & 34,2 & 68,4 & 40,1 \\
\hline $\begin{array}{c}\text { SVM- } \\
\text { Sigmoid }\end{array}$ & & 27,8 & 58,9 & 34,2 & 67,6 & 40,8 \\
\hline Isodata & & 37,2 & 78,7 & 47,9 & 64,6 & 46,4 \\
\hline $\mathrm{ML}$ & Suelo 2 & 58,8 & 128,1 & 78,8 & 92,3 & 43,4 \\
\hline SVM-L & & 62,6 & 136,8 & 83 & 93,3 & 43,9 \\
\hline SVM-P & & 62,4 & 136,4 & 82,7 & 92,9 & 44,9 \\
\hline SVM-RBF & & 62,4 & 136,4 & 82,7 & 92,9 & 44,7 \\
\hline $\begin{array}{c}\text { SVM- } \\
\text { Sigmoid }\end{array}$ & & 62 & 135,4 & 82 & 92,2 & 45,1 \\
\hline Isodata & & 60,8 & 131,6 & 79,2 & 87,6 & 53,4 \\
\hline ML & Suelo3 & 49 & 107,5 & 65,6 & 79,1 & 42,6 \\
\hline SVM-L & & 46,3 & 100,9 & 61,7 & 77,2 & 44,5 \\
\hline SVM-P & & 46,4 & 101 & 61,8 & 77,2 & 44,6 \\
\hline SVM-RBF & & 46,4 & 101 & 61,8 & 77,2 & 44,7 \\
\hline $\begin{array}{c}\text { SVM- } \\
\text { Sigmoid } \\
\end{array}$ & & 46,3 & 100,8 & 61,7 & 76,9 & 44,8 \\
\hline ISOC & & 50,5 & 109,9 & 67,1 & 79,8 & 40,2 \\
\hline $\mathrm{ML}$ & Suelo 4 & 66,8 & 147,4 & 90 & 97,8 & 46,6 \\
\hline SVM-L & & 74,6 & 163 & 97,7 & 103,7 & 55,7 \\
\hline SVM-P & & 75,7 & 165 & 98,6 & 104,4 & 55,5 \\
\hline SVM-RBF & & 75,5 & 164,5 & 98,4 & 104,2 & 55,5 \\
\hline $\begin{array}{c}\text { SVM- } \\
\text { Sigmoid }\end{array}$ & & 75,2 & 164,2 & 98,4 & 104,4 & 54 \\
\hline Isodata & & 71,6 & 155,8 & 93,6 & 99,6 & 47,1 \\
\hline ML & Urbano 1 & 33,8 & 71,7 & 48,5 & 53,2 & 109,9 \\
\hline SVM-L & & 32,3 & 72,5 & 55,9 & 67,6 & 142,5 \\
\hline SVM-P & & 32,3 & 72,2 & 54,5 & 67,3 & 135,6 \\
\hline SVM-RBF & & 32,6 & 72,6 & 53,8 & 67 & 132,2 \\
\hline $\begin{array}{c}\text { SVM- } \\
\text { Sigmoid }\end{array}$ & & 32,7 & 73 & 55,4 & 66,1 & 147,7 \\
\hline ISOC & & 33,9 & 72,4 & 50,6 & 59,6 & 163,4 \\
\hline $\mathrm{ML}$ & Urbano 2 & 38 & 81,4 & 52 & 61,6 & 106 \\
\hline SVM-L & & 36,7 & 75,8 & 47,1 & 52,7 & 142,7 \\
\hline SVM-P & & 36,2 & 75,2 & 47,5 & 53,2 & 141,7 \\
\hline SVM-RBF & & 36,2 & 75,3 & 47,9 & 53,6 & 141,2 \\
\hline $\begin{array}{c}\text { SVM- } \\
\text { Sigmoid }\end{array}$ & & 36,1 & 73,7 & 45,1 & 50 & 142,4 \\
\hline Isodata & & 39,4 & 77,7 & 45,4 & 50,3 & 94,3 \\
\hline ML & Urbano 3 & 45 & 95 & 56,5 & 60,3 & 63,7 \\
\hline SVM-L & & 43,6 & 93,3 & 56,7 & 68,4 & 75,5 \\
\hline SVM-P & & 45,3 & 97,1 & 58,7 & 70,8 & 77 \\
\hline SVM-RBF & & 45,4 & 97,4 & 58,9 & 70,8 & 76,8 \\
\hline $\begin{array}{c}\text { SVM- } \\
\text { Sigmoid }\end{array}$ & & 45,1 & 96,6 & 58,5 & 70,6 & 76,6 \\
\hline Isodata & & - & - & - & - & - \\
\hline ML & Urbano 4 & 88 & 173,7 & 98,8 & 97,8 & 69,2 \\
\hline SVM-L & & 113,5 & 203,7 & 117,5 & 112,8 & 75,6 \\
\hline
\end{tabular}




\begin{tabular}{|c|c|c|c|c|c|c|}
\hline SVM-P & & 116,5 & 206,7 & 119,6 & 114,3 & 78,4 \\
\hline SVM-RBF & & 116,6 & 206,4 & 119,4 & 114 & 76,8 \\
\hline $\begin{array}{c}\text { SVM- } \\
\text { Sigmoid }\end{array}$ & & 111,7 & 203,3 & 117,1 & 113,1 & 76,5 \\
\hline Isodata & & 98 & 193 & 111,9 & 113,5 & 74,4 \\
\hline ML & Veg. 1 & 28,7 & 59,7 & 33,6 & 62,5 & 81,3 \\
\hline SVM-L & & 23 & 49,2 & 27 & 62,1 & 104,4 \\
\hline SVM-P & & 22,9 & 49 & 26,9 & 62 & 103,8 \\
\hline SVM-RBF & & 23,1 & 49,2 & 27,1 & 61,3 & 103,6 \\
\hline $\begin{array}{c}\text { SVM- } \\
\text { Sigmoid }\end{array}$ & & 22,4 & 47,9 & 26,3 & 61,3 & 110,7 \\
\hline Isodata & & 16,8 & 31,2 & 18,7 & 31 & 127 \\
\hline ML & Veg. 2 & 15,8 & 30,1 & 15,5 & 48,5 & 83,5 \\
\hline SVM-L & & 18,2 & 37,3 & 18,2 & 72,9 & 84,6 \\
\hline SVM-P & & 18,2 & 37,3 & 18,2 & 71,7 & 83,7 \\
\hline SVM-RBF & & 18,2 & 37,3 & 18,2 & 71,7 & 84,7 \\
\hline $\begin{array}{c}\text { SVM- } \\
\text { Sigmoid }\end{array}$ & & 18,6 & 38,4 & 18,9 & 71,4 & 79,7 \\
\hline ISOC & & 18,3 & 38,1 & 18,8 & 72,4 & 95,8 \\
\hline ML & Vías 1 & 58,1 & 113,8 & 63,7 & 66 & 93,6 \\
\hline SVM-L & & 63 & 122,7 & 67,4 & 69,6 & 94 \\
\hline SVM-P & & 62,6 & 121,4 & 66,6 & 68,6 & 94,1 \\
\hline SVM-RBF & & 62,8 & 122 & 67 & 69,1 & 94,4 \\
\hline $\begin{array}{c}\text { SVM- } \\
\text { Sigmoid }\end{array}$ & & 62,5 & 121,8 & 67 & 70 & 94,8 \\
\hline Isodata & & 62,5 & 125,5 & 69,9 & 76,2 & 102,7 \\
\hline ML & Vías 2 & 52 & 102,6 & 57,8 & 59,8 & 67,5 \\
\hline SVM-L & & 47 & 89,8 & 49,6 & 50,7 & 69,9 \\
\hline SVM-P & & 45,1 & 86 & 47,8 & 49,2 & 68,8 \\
\hline SVM-RBF & & 45 & 85,9 & 47,7 & 49 & 68,6 \\
\hline $\begin{array}{c}\text { SVM- } \\
\text { Sigmoid }\end{array}$ & & 42,9 & 82,1 & 45,9 & 47,9 & 69,5 \\
\hline Isodata & & 46,9 & 98,7 & 59,2 & 69,8 & 69,3 \\
\hline ML & Río & 21,8 & 48,6 & 23,8 & 17 & 13,8 \\
\hline SVM-L & & 13,9 & 22,5 & 12 & 18,5 & 14,9 \\
\hline SVM-P & & 23,3 & 51,2 & 25,4 & 19,6 & 13,9 \\
\hline SVM-RBF & & 13,9 & 22,3 & 11,9 & 18 & 15,3 \\
\hline $\begin{array}{c}\text { SVM- } \\
\text { Sigmoid }\end{array}$ & & 23,1 & 50,8 & 25,1 & 19,2 & 13,9 \\
\hline Isodata & & 22,2 & 48,6 & 24 & 18,8 & 14 \\
\hline ML & Sombra & 13,6 & 21,6 & 11,4 & 11,7 & 12,9 \\
\hline SVM-L & en río & 13,9 & 22,5 & 12 & 18,5 & 14,9 \\
\hline SVM-P & & 13,9 & 22,3 & 11,9 & 18,1 & 15,3 \\
\hline SVM-RBF & & 13,9 & 22,3 & 11,9 & 18 & 15,3 \\
\hline $\begin{array}{c}\text { SVM- } \\
\text { Sigmoid }\end{array}$ & & 13,8 & 22,1 & 11,9 & 17,9 & 15,6 \\
\hline ISOC & & - & - & - & - & - \\
\hline ML & Sombra & 13 & 18,2 & 10,2 & 12 & 62,3 \\
\hline SVM-L & en suelo & 13,9 & 22,4 & 13,1 & 18,9 & 84,6 \\
\hline SVM-P & & 14 & 22,4 & 13,2 & 19 & 83,7 \\
\hline SVM-RBF & & 13,9 & 22,4 & 13,1 & 18,9 & 83,9 \\
\hline $\begin{array}{c}\text { SVM- } \\
\text { Sigmoid }\end{array}$ & & 13,9 & 22,1 & 13 & 18,7 & 81,4 \\
\hline Isodata & & 14 & 21,7 & 12,6 & 18,3 & 65,2 \\
\hline
\end{tabular}

\section{AGRADECIMIENTOS}

Los autores reconocen las contribuciones de Alfonso Andrés Urrutia (Inclam S. A.), Gonzalo Tentor, Tomás Fernández de Sevilla y Francisco J. Arjonilla (Stereocarto S.L.) por su colaboración y cesión de la imagen utilizada.

\section{REFERENCIAS}

[1] R. A. Fowler (2000), "The lowdown on LIDAR". Earth Observation Magazine, 19. Disponible en:

http://www.eomonline.com/Common/Archives/2000mar/00mar_fowler. html

[2] M. E. Hodgson , J. R.Jensen, L. Schmidt, S., Schill and B. Davis "An evaluation of LIDAR- and IFSAR-derived digital elevation models in leaf on conditions with USGS Level 1 and Level 2 DEMs". Remote Sensing of Environment, 84, pp. 295-308, 2003.

[3] D. S., Lee and J. Shan. "Combining LIDAR elevation data and IKONOS multispectral imagery for coastal classification mapping". Marine Geodesy, 26, pp. 117-127. 2003.

[4] E.W. Bork and J.G. Su, "Integrating LIDAR data and multispectral imagery for enhanced classification of rangeland vegetation: A meta analysis", Remote Sensing of Environment, 111, pp. 11-24, 2007.

[5] R. Brennan and T. L. Webster, "Object-oriented land cover classification of LIDAR-derived surfaces," Can. J. Remote Sens., vol. 32, no. 2, pp. 162-172, 2006.

[6] A. P. Charaniya, R. Manduchi, and S. K. Lodha, "Supervised parametric classification of aerial LiDAR data," in Proc. IEEE Comput. Soc. Conf. CVPRW, 2004, p. 30.

[7] J. Holmgren and $\AA$. Persson, "Identifying species of individual trees using airborne laser scanner," Remote Sens. Environ., vol. 90, no. 4, pp. 415-423, Apr. 2004.

[8] D. Lemp and U. Weidner, "Improvements of roof surface classification using hyperspectral and laser scanning data," in Proc. ISPRS Joint Conf.: 3rd Int. Symp. Remote Sens. Data Fusion Over Urban Areas (URBAN), 5th Int. Symp. Remote Sens. Urban Areas (URS), Tempe, AZ, Mar. 14-16, 2005.

[9] E. M. Perry, H. P. Foote, G. M. Petrie, K. L. Steinmaus, D. E. Irwin, and A. J. Stephan, "Exploitation of hyperspectral imagery and Lidar for land use classification," in Proc. IGARSS, Seattle,WA, 1998, pp. 10131015.

[10] M. Dalponte, L. Bruzzone and D. Gianelle, "Fusion of Hyperspectral and LIDAR Remote Sensing Data for Classification of Complex Forest Areas", IEEE Transactions on Geoscience and Remote Sensing, Vol. 46, No. 5, May 2008.

[11] M. Bartels and H. Wei. "Rule-based improvement of maximum likelihood classified LIDAR data fused with co-registered bands". Annual Conference of the Remote Sensing and Photogrammetry Society, CD Proceedings, 05-08 September 2006, pp. 1-9.

[12] A.S. Antonarakis, K.S. Richards and J. Brasington, "Object-based land cover classification using airborne LiDAR", Remote Sensing of Environment, 112, pp. 2988-2998, 2008.

[13] M. Mutlu, S. C. Popescu, C. Stripling and T. Spencer, "Mapping surface fuel models using lidar and multispectral data fusion for fire behaviour", Remote Sensing of Environment 112, pp. 274-285, 2008.

[14] A. Arquero, E. Martínez, C. Gonzalo y A. Ferreras. "Utilización de scattergrams de ErMapper 5.5 para la elección de áreas de entrenamiento en la clasificación temática de imágenes Landsat", Mapping, vol. 50, pp. 56-58, 1998.

[15] J. M. Vázquez, A. Arquero y E. Martínez, " Application of statistical models to the efficient selection of endmembers by Field Radiometry", IEEE Latin America Transactions, Vol. 5, No. 8, pp. 561-567, Dec. 2007.

[16] P. M., Mather, Computer Processing of Remotely-Sensed Images. An Introduction, Wiley, 2004.

[17] V. Vapnik, The nature of statistical learning theory, Springer- Verlag New York, Inc, 1995.

[18] G. M. Foody and A. Mathur. "A Relative Evaluation of Multiclass Image Classification by Support Vector Machines". IEEE Transactions on Geoscience and Remote Sensing, 42 (6), pp. 1335-1343, 2004.

[19] H. Chia, L. T Deepu Rajan Liu, S. and Yi. "Adaptive hierarchical multiclass SVM classifier for texture-based image classification", in Multimedia and Expo, 2005. ICME 2005. IEEE International Conference, p. 4, 6-8, July 2005. 
[20] Stereocarto S. L., http://www.stereocarto.com/es/productos/producto.php?id=10

[21] L.T. Waser, E. Baltsavias, K. Ecker, H. Eisenbeiss, E. FeldmeyerChriste, C. Ginzler, M. Küchler, L. Zhang, "Assessing changes of orest area and shrub encroachment in a mire ecosystem using digital surface models and CIR aerial images", Remote Sensing of Environment 112, pp. 1956-1968, 2008.

[22] E. Martínez, A. Arquero, C. Gonzalo y M. Lillo, "Control de la calidad de imágenes fusionadas para la clasificación temática de zonas espacial y/o espectralmente confusas" en TELEDETECCIÓN. Herramienta para la gestión sostenible, Raúl Rivas, Alfredo Grisotto y Mónica Sacido (Eds.), Mar de Plata (Argentina), 2007, pp.445-453.

[23] J.T. Tou and R.C. Gonzalez, Pattern Recognition Principles, AddisonWesley, Reading. 1974.

[24] C. Liu, P. Frazier and L. Kumar, "Comparative assessment of the measures of thematic classification accuracy", Rem. Sen. of Environment, 107, pp. 606-616, 2007.

[25] A. Arquero, M. Álvarez y E. Martínez, "Decision Management Making by AHP (Analytical Hierarchy process) through GIS data", IEEE Latin America Transactions, Vol. 7, No. 1, pp. 101-106, March 2009.

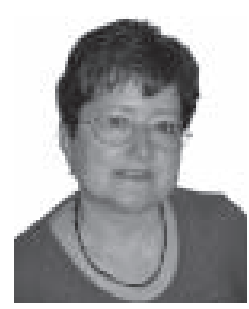

Águeda Arquero Hidalgo, obtuvo el Grado de Doctor en Ciencias Químicas por la Universidad Autónoma de Madrid (España) en 1983 Actualmente es Profesora Titular de Universidad en el Departamento de Arquitectura y Tecnología de Sistemas Informáticos, en la Facultad de Informática de la Universidad Politécnica de Madrid. Su interés en la investigación en el campo del procesado y análisis de imágenes remotamente detectadas incluye la medición de la respuesta radiométrica de cubiertas terrestres in situ y su procesado posterior; el análisis de datos teledetectados usando algoritmos de redes neuronales artificiales, algoritmos genéticos y lógica difusa. Otros campos de investigación de su interés son la síntesis y caracterización espectral de nuevos materiales. Es miembro de la Asociación Española de Teledetección.

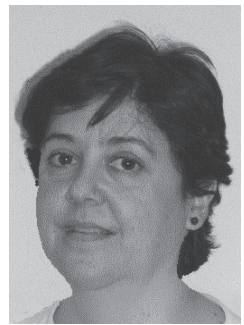

Estíbaliz Martínez Izquierdo obtuvo el Grado de Doctor en Ciencias Químicas por la Universidad Complutense de Madrid (España) en 1989. Actualmente es Profesora Titular de Universidad en el Departamento de Arquitectura y Tecnología de Sistemas Informáticos de la Facultad de Informática de la Universidad Politécnica de Madrid (España). Imparte conocimientos en Diseño Digital,Tecnología VLSI y Teledetección. Sus intereses en investigación están relacionados con las áreas del procesado y análisis de imágenes en Teledetección, incluyendo la utilización de algoritmos basados en Redes Neuronales, Genéticos y Lógica Fuzzy para el análisis e interpretación de los datos captados desde sensores situados en plataformas aero-espaciales. Además dentro de sus líneas de investigación se incluyen el diseño e implementación de sistemas de almacenamiento y gestión de la información de Teledetección mediante acceso a arquitecturas cliente-servidor. 